Kurztitel: CBM BEI PTBS - Running title: CBM FOR PTSD

\title{
Cognitive Bias Modification bei Symptomen der Posttraumatischen Belastungsstörung (PTBS)
}

\section{Cognitive Bias Modification for Symptoms of Posttraumatic Stress Disorder (PTSD)}

\author{
Marcella L. Woud ${ }^{\mathrm{a}}$ \\ Charlotte E. Wittekind ${ }^{\mathrm{b}}$ \\ Felix Würtz ${ }^{\mathrm{a}}$
}

a. Mental Health Research and Treatment Center, Department of Psychology, Ruhr-Universität Bochum, Massenbergstrasse 9-13, 44787 Bochum, Germany. Tel.: +49 (0) 2343221502. E-mail: Marcella.Woud@rub.de

b. Department of Psychology, LMU Munich, Leopoldstraße 13, 80802 Munich, Germany

Corresponding author: Marcella L. Woud, Mental Health Research and Treatment Center, Department of Psychology, Ruhr-Universität Bochum, Massenbergstrasse 9-13, 44787 Bochum, Germany. E-mail: marcella.woud@ @ub.de. Tel.: +49 (0) 2343221502. Fax: +49 (0) 2343214369.

Keywords Deutsch: Cognitive Bias Modification; Kognitive Verzerrungen; Posttraumatische Belastungsstörung

Keywords Englisch: Cognitive Bias Modification; Cognitive Biases; Posttraumatic Stress

Disorder 


\begin{abstract}
Deutsch
Kognitive Modelle der Posttraumatischen Belastungsstörung (PTBS) gehen davon aus, dass eine dysfunktionale Informationsverarbeitung in den Bereichen Aufmerksamkeit, Interpretation, Bewertung und Gedächtnis einen wichtigen Faktor für das Auftreten und die Aufrechterhaltung der PTBS darstellt. Parallel zeigen verschiedenste Entwicklungen in der klinisch-experimentellen Forschung, dass es möglich sein könnte, solche kognitiven Verzerrungen mit Hilfe von Cognitive Bias Modification (CBM)-Trainings zu modifizieren. In diesem Beitrag geben wir einen narrativen Überblick über die CBM-Forschung im Kontext von Trauma und PTBS, im experimentellen sowie klinisch-angewandten Bereich. Zudem werden Herausforderungen und neue Forschungslinien für die CBM-Forschung im Kontext der PTBS vorgestellt und diskutiert.
\end{abstract}

\footnotetext{
Abstract Englisch

Cognitive models of Posttraumatic Stress Disorder (PTSD) postulate that dysfunctional processing biases in the contexts of attention, interpretation, appraisal, and memory to be a crucial factor in the development and maintenance of PTSD. Parallel, advances in the field of experimental psychopathologies suggest that it could be possible to modify these cognitive biases via Cognitive Bias Modification (CBM)-trainings. In this article we narratively review the current literature on CBM research in the context of trauma and PTSD, across both experimental and clinical research settings. In addition, we discuss current challenges and future directions for CBM research in the context of PTSD.
} 
Die Posttraumatische Belastungsstörung (PTBS) ist eine psychologische Reaktion, die bei Personen auftreten kann, die mit einem Trauma konfrontiert wurden, beispielsweise einem schweren Unfall oder Gewalt. Die PTBS geht mit verschiedensten Symptomen einher (DSM-5, American Psychiatric Association, 2013): (a) unwillkürliche Erinnerungen an das Trauma, z.B. in Form von Flashbacks; (b) Vermeidung von Reizen, die mit dem Trauma assoziiert sind; (c) negative Veränderungen in Kognitionen und Stimmung; und (d) negative Veränderungen des Erregungsniveaus und der Reaktivität. Verschiedene kognitive Theorien schreiben der Informationsverarbeitung eine zentrale Rolle für die Entwicklung und Aufrechterhaltung der PTBS zu (z.B. Brewin, 2014; Dalgleish, 2004; Ehlers \& Clark, 2000; Foa et al., 1989), vor allem in den Bereichen Aufmerksamkeit, Bewertung und Interpretation und Gedächtnis ${ }^{1}$. Diese Modelle postulieren, dass PTBS-Patient*innen dazu neigen, traumarelevante Reize vorranging und vor allem negativ zu verarbeiten. Es konnte hierbei unter anderem gezeigt werden, dass PTBS-Patient*innen ihre Aufmerksamkeit schneller auf bedrohliche als auf neutrale Reize richten. Zudem bewerten und interpretieren PTBS-Patient*innen traumarelevante Reize eher als bedrohlich bzw. zeigen einen verstärkten Abruf von traumabezogenen versus nichttraumabezogenen Erinnerungen (für Übersichtsarbeiten, siehe Bomyea et al., 2017; Woud et al., 2017). Zudem gibt es zahlreiche Belege dafür, dass eine dysfunktionale Informationsverarbeitung die Entwicklung von PTBS-Symptomen vorhersagt (z.B. Bryant \& Guthrie, 2007; Imbriano et al., 2022).

Aufgrund des Themas dieses Artikels möchten wir uns nun jedoch auf die wachsende Anzahl an Studien richten, die sich auf die Modifikation der dysfunktionalen Informationsverarbeitung und deren Effekte auf (analoge) PTBS-Symptome fokussieren. Diese Verfahren beinhalten systematische, computergestützte Trainings, die überwiegend im Rahmen

\footnotetext{
${ }^{1}$ In der emotionalen Psychopathologie werden drei klassische kognitive Verzerrungen thematisiert - Verzerrungen in der Aufmerksamkeit, der Interpretation und des Gedächtnisses (Mathews \& MacLeod, 2005). Im Bereich der PTBS sind alle drei Verzerrungen relevant und mit ihr assoziiert, jedoch gibt es wenige Studien zu den klassischen Interpretationsverzerrungen, dafür jedoch viele zu dysfunktionalen Bewertungen. Auch wenn sich Interpretationsund Bewertungsprozesse ähneln, z.B. beide involvieren evaluierende Aspekte, möchten wir diese Prozesse getrennt betrachten, und zwar aufgrund ihrer Operationalisierung in den jeweiligen Trainings, hier am Beispiel positiver Trainings: Bei Interpretationstrainings werden ambige Reize/Szenarios verwendet, und Teilnehmende trainieren hierbei, diese ambigen Reize/Szenarios funktional zu interpretieren (z.B., dass sich eine potenziell bedrohliche Situation als ungefährlich entpuppt). Bei Bewertungstrainings hingegen werden Reaktionen auf das (analoge) Trauma und die eigenen Symptome als Reize/Szenarios verwendet, und hier trainieren Teilnehmende, diese Reaktionen und Symptome funktional zu bewerten (z.B., dass Intrusionen ein normaler Teil der Verarbeitung sind). Beim Beschreiben der Modifikationsstudien werden wir somit getrennt auf Bewertungs- bzw. Interpretationstrainings eingehen. Des Weiteren nutzen wir den Term ,dysfunktionale Informationsverarbeitung“, um die relevanten kognitiven Prozesse so generisch wie möglich darzustellen.
} 
Kurztitel: CBM BEI PTBS - Running title: CBM FOR PTSD

der Cognitive Bias Modification (CBM)-Forschung entwickelt wurden (Koster et al., 2009; Woud \& Becker, 2014). Um eine Veränderung der verzerrten Informationsverarbeitung zu erreichen, wurden Computeraufgaben, die ursprünglich zur Messung von dysfunktionalen Prozessen eingesetzt wurden, in Trainingsparadigmen umgewandelt. Dies bedeutet konkret, dass Teilnehmende instruiert wurden, systematisch eine vordefinierte Reaktion (z.B. eine positive und funktionale) zu den im Training angebotenen, traumarelevanten Reizen abzugeben. Die zugrundeliegende Idee hierbei ist, dass eine sogenannte „Kontingenz“ zwischen störungsrelevanten Reizen und den ausgeführten Reaktionen hergestellt wird, und dass dadurch eine Veränderung der Informationsverarbeitung eintritt, die sich dann auch auf der Symptomebene auswirkt. Ein Beispiel im Rahmen der Modifikation dysfunktionaler Bewertungen zur Verdeutlichung: Stellen Sie sich eine Person vor, die in Folge eines Traumas PTBS-Symptome entwickelt hat und damit einhergehende dysfunktionale Bewertungen, z.B. "Ich werde das Trauma nie verarbeiten können“. In einer CBM-Trainingsstudie würden nun Szenarios, d.h. kurze Beschreibungen traumarelevanter Reaktionen oder (Reaktionen auf) Symptome, angeboten, die genau diese Bewertungen aufgreifen. Die Trainingsszenarios haben jedoch kein Ende und es ist die Aufgabe der Teilnehmenden, die Szenarios zu beenden. Dies geschieht, indem die Teilnehmenden Wortfragmente, die ihnen nach jedem Szenario angeboten werden, vervollständigen. Ein Beispiel: „Die Tatsache, dass ich plötzlich an das Ereignis denken muss, bedeutet, dass ich es ... ve_rbeite“; Lösung: „,verarbeite“; komplettes Szenario: „Die Tatsache, dass ich häufig und plötzlich an das Ereignis denken muss, bedeutet, dass ich es verarbeite.“ Bei diesem Beispiel wurde ein positives Wortfragment eingesetzt, und durch das Vervollständigen dieses Wortfragments wird das Szenario somit auf eine positive Weise beendet bzw. werden die Reaktionen auf die Intrusionen als funktional bewertet. Es wird erwartet, dass sich im Verlauf des Trainings eine Kontingenz zwischen traumarelevanten Szenarios und funktionalen Bewertungen entwickelt, die sich immer weiter verstärkt. Konkret bedeutet dies, dass traumarelevante Informationen nicht mehr negativ oder bedrohlich, sondern neutral bzw. funktional bewertet werden, und dass sich dies positiv auf der Symptomebene auswirkt.

Nach MacLeod und Mathews (2012) ist die CBM-Forschung durch drei Hauptziele motiviert, nämlich (1.) die potenziell kausale Rolle der dysfunktionalen Informationsverarbeitung bei der Entstehung und Aufrechterhaltung psychopathologischer Symptome zu untersuchen; (2.) unser Verständnis der zugrundeliegenden Mechanismen klinisch-relevanter, kognitiver Prozesse zu verbessern, und (3.) zu testen, ob CBM-Verfahren einen therapeutischen Nutzen haben (siehe 
Kurztitel: CBM BEI PTBS - Running title: CBM FOR PTSD

auch Gober et al., 2021). Das CBM-Forschungsfeld steckt generell noch in den Kinderschuhen und ist mit verschiedensten Herausforderungen konfrontiert (vgl. Fodor et al., 2020; Jones \& Sharpe, 2017), trotzdem gibt es immer mehr Belege dafür, dass CBM-Verfahren vielversprechende Effekte auf (analoge) PTBS-Symptome haben. In den folgenden Abschnitten geben wir einen narrativen Überblick über die klinische und experimentelle CBM-Forschung im Kontext der PTBS bzw. von Traumata. Experimentelle Studien verwenden dabei häufig sogenannte ,,analoge Traumata“. Analoge Traumata beschreiben hierbei Verfahren oder Situationen, die nicht den Kriterien eines Traumas entsprechen und die in ethischer Weise im Labor verwendet werden können, aber dennoch Symptome aus dem Bereich der PTBS hervorrufen, wie zum Beispiel belastende Intrusionen (z.B. Trauma-Film-Paradigma; James et al., 2016) (für eine Diskussion zum Übergang von experimentellen zu klinischen Studien siehe Blackwell \& Woud, 2022).

\section{Modifikation von Aufmerksamkeitsprozessen}

Die Studie von Verwoerd et al. (2012) ist eine der ersten Studien, in denen die Effekte eines sogenannten Attention Bias Modification (ABM)-Trainings getestet wurden. Hierbei wurden gesunde Studierende untersucht, denen als analoges Trauma verschiedene belastende Filmclips gezeigt wurden (Trauma-Film-Paradigma). Als Training wurde eine modifizierte „spatial cuing task“ (Posner, 1980) verwendet, welche zwei Arten von Stimuli enthielt: Fotos mit Szenen aus den Trauma-Filmen sowie neutrale Kontrollbilder. In der aktiven Bedingung wurden die Teilnehmenden trainiert, ihre Aufmerksamkeit systematisch von den Fotos aus den TraumaFilmen loszulösen, indem sie einen Buchstaben identifizieren mussten, der an anderer Stelle dargeboten wurde. In der Kontrollbedingung wurde die Aufmerksamkeit nicht in eine bestimmte Richtung trainiert. Die Ergebnisse zeigten, dass Teilnehmende aus der aktiven Trainingsbedingung weniger durch Fotos aus den Trauma-Filmen abgelenkt wurden und dass sie weniger filmbezogene Intrusionen erlebten, verglichen mit Kontrollpersonen.

Die Dot-Probe-Aufgabe (MacLeod et al., 1986) ist zurzeit das am häufigsten verwendete Paradigma im Kontext von ABM, bei dem Teilnehmende trainieren, ihre Aufmerksamkeit auf neutrale und nicht auf bedrohliche Stimuli zu richten. Die Dot-Probe-Aufgabe wird im TraumaKontext vor allem im klinisch-angewandten Bereich eingesetzt, entweder als eigenständige Intervention oder als Zusatz zu einer etablierten Traumatherapie. Die erste Studie war die randomisiert-kontrollierte Studie von Schoorl et al. (2013), welche den Effekt von ABM bei 
Kurztitel: CBM BEI PTBS - Running title: CBM FOR PTSD

PTBS-Patient*innen untersuchte, die auf einen Therapieplatz warteten. Im aktiven ABMTraining wurde die Aufmerksamkeit weg von bedrohlichen und hin zu neutralen Stimuli trainiert, wohingegen in der Kontrollbedingung trainiert wurde, die Aufmerksamkeit gleich oft auf neutrale wie auf bedrohliche Reize zu richten. Entgegen den Erwartungen führten sowohl das aktive ABM- als auch das Kontrolltraining zu einer Verringerung der PTBS-Symptome (zu ähnlichen Befunden siehe Khanna et al., 2016). In der Studie von Kuckertz et al. (2014) wurde das ABM-Training als ergänzende Maßnahme zur traumafokussierten PTBS-Behandlung eingesetzt und Patient*innen trainierten, ihre Aufmerksamkeit von bedrohlichen Reizen zu lösen. Patient*innen in der Kontrollbedingung richteten ihre Aufmerksamkeit gleich oft auf bedrohliche und neutrale Reize. Die Ergebnisse zeigten, dass ABM, im Vergleich zur Kontrollbedingung, zu einer signifikanten Verringerung der Aufmerksamkeitsverzerrungen führte, jedoch nur wenn die Messung der Aufmerksamkeitsverzerrung neue Stimuli enthielt. Bezüglich der Symptomatik wurde gefunden, dass ABM, im Vergleich zur Kontrollbedingung, auch zu einer Verringerung in allgemeinen Symptomen führte (z.B. Depression). Diese ersten Studien führten aufgrund der Ergebnisse zu einer neuen Forschungslinie im Bereich der PTBS, nämlich bezüglich der Kontrollbedingungen: Generell soll die eingesetzte Kontrollbedingung keine Trainingskontingenz beinhalten, also die Aufmerksamkeitsausrichtung nicht systematisch trainieren, da in diesen Bedingungen die Aufmerksamkeit gleich oft auf neutrale sowie bedrohliche Reize gerichtet werden sollte. Dennoch könnten solche Bedingungen als eine niedrigdosierte Version des aktiven ABM-Trainings angesehen werden beziehungsweise andere kognitive Prozesse trainieren, die im Bereich der PTBS relevant sind, unter anderem Aufmerksamkeitskontrolle. Dies könnte erklären, warum die eingesetzten Kontrollbedingungen im Grunde eine Variante einer aktiven Bedingung sein könnten. Die Studien von Badura-Brack et al. (2015) und Lazarov et al. (2019) sind dieser Hypothese nachgegangen und verglichen die Effekte von ABM und Aufmerksamkeitskontrolltrainings als eigenständige Intervention bei PTBS-Patient*innen. In beiden Studien konnte gezeigt werden, dass sowohl ABM als auch das Aufmerksamkeitskontrolltraining einen positiven Einfluss auf die Reduktion der Aufmerksamkeitsverzerrungen sowie der PTBS-Symptome ausübten. Bezüglich der PTBSSymptomatik schien das Aufmerksamkeitskontrolltraining dem klassischen ABM-Training jedoch leicht überlegen zu sein. Eine aktuelle Studie von Segal et al. (2020) untersuchte, inwieweit ein personalisiertes Aufmerksamkeitskontrolltraining die Trainingseffekte noch weiter verstärken kann, indem Reize verwendet wurden, die die Patient*innen vorab als besonders 
Kurztitel: CBM BEI PTBS - Running title: CBM FOR PTSD

bedrohlich bewertet hatten. Das personalisierte Training wurde mit der Standardversion des Aufmerksamkeitskontrolltrainings sowie einer Kontrollbedingung verglichen, das den jeweiligen aktiven Trainings von der Operationalisierung ähnlich war, jedoch keine der potenziell aktiven Mechanismen enthielt (die Aufgabe der Teilnehmenden in der Kontrollbedingung war es, Buchstaben, die auf neutrale Wörter folgten zu identifizieren). Die Stichprobe bestand aus diagnostizierten PTBS-Patient*innen von denen die meisten keine systematische therapeutische Unterstützung erhielten. Beide Versionen des Aufmerksamkeitskontrolltrainings führten zu einer Reduktion der Aufmerksamkeitsverzerrungen, nicht jedoch die Kontrollbedingung. Entgegen den Erwartungen konnte jedoch bei allen drei Trainings ein positiver Effekt auf die PTBS- sowie die depressive Symptomatik beobachtet werden (siehe Segal et al., 2020 zu einer ähnlichen Studie im Bereich der Akuten Belastungsstörung).

Die klassischen Dot-Probe-Trainings, die bis jetzt vorgestellt wurden, wurden vor dem Hintergrund der Annahmen kognitiver Modelle entwickelt: Die PTBS ist durch verzerrte Aufmerksamkeitsprozesse hin zu bedrohlichen Reizen gekennzeíchnet, folglich sollte eine Reduktion der Verzerrung mit einer Abnahme der Symptomatik assoziiert sein (Clarke et al., 2014). Das Weglenken der Aufmerksamkeit von bedrohlichen Reizen war somit zentrales Element initialer Trainingsstudien. Aus klinischer Perspektive steht so ein Ansatz jedoch im Widerspruch zu unseren evidenzbasierten therapeutischen Interventionen, bei denen vor allem die Konfrontation mit Trauma-assoziierten Reizen zentraler Bestandteil der Behandlung ist. Die Studie von Wald et al. (2016) war eine der ersten Studien, die diesen Aspekt berücksichtigte und untersuchte, inwieweit ABM, das die Aufmerksamkeit hin zu bedrohlichen Reizen trainiert, einen präventiven Effekt hat, Das Training wurde gesunden Soldaten vor ihrem Kriegseinsatz angeboten und dessen Effekte mit einem Aufmerksamkeitskontrolltraining sowie einer Kontrollbedingung, die kein Training erhielt, verglichen. Die Ergebnisse zeigten, dass ABM, im Vergleich zu den anderen beiden Bedingungen, das Risiko, nach dem Einsatz eine PTBS zu entwickeln, signifikant reduzierte (zu ähnlichen Befunden siehe Wald et al., 2017). Zwischenfazit: ABM-Trainings haben in den letzten Jahren eine große Bandbreite an Forschungslinien hervorgebracht, die klinischen Studien weisen jedoch ein heterogenes Bild auf. Des Weiteren führten teilweise auch Kontrollbedingungen, die keine der postulierten Mechanismen trainierten, zu einer Verbesserung der Symptomatik (z.B. Schoorl et al., 2013; Segal et al., 2020), somit bedarf es systematischer Nachfolgeuntersuchungen, um die inkonsistenten Ergebnisse erklären zu können. 
Kurztitel: CBM BEI PTBS - Running title: CBM FOR PTSD

\section{Modifikation von Bewertungs- und Interpretationsprozessen}

Im Bereich der Trainings zur Modifikation dysfunktionaler Bewertungen und Interpretation gibt eine große Anzahl an experimentellen Laborstudien, dafür jedoch weniger klinische Studien. Im Bereich der PTBS steht vor allem die Veränderung von

Bewertungsprozessen mittels sogenannter ,(re)appraisal trainings“ im Fokus. Zentral hierbei sind die Modifikation von Bewertungsprozessen bezüglich des Trauma und der (analogen) PTBS-

Symptome. Eine der ersten Trainingsstudien in diesem Bereich sind die Arbeiten von Schartau et al. (2009). Alle Studien umfassten gesunde Teilnehmende und verwendeten sowohl nichtautobiografische als auch autobiografische belastende Erinnerungen (z.B. mittels Trauma-FilmParadigma bzw. es wurden Teilnehmende eingeladen, die ein belastendes autobiografisches Ereignis erlebt hatten). In den Studien wurden allgemein gültige beziehungsweise funktionale Bewertungen eingesetzt, beispielsweise „In der Welt passieren schlechte Dinge, ich muss diese hinter mir lassen und weitermachen“ oder „Jede Situation hat in der Regel auch ihre guten Seiten, und es ist wichtig, sich auf diese zu konzentrieren“. Für das Training bedeutete das, dass die Teilnehmenden diese Bewertungsregeln anwenden sollten, während sie sich beispielsweise die Trauma-Filme ansahen oder an ihr eigenes belastendes Ereignis zurückdachten. Die Effekte dieses Trainings wurden dann mit den Effekten verschiedener Kontrollbedingungen verglichen (z.B. Gruppen, die ihren Emotionen freien Lauf ließen oder sich auf technische Aspekte der Trauma-Filme fokussieren sollten). Über alle Studien hinweg wurden positive Trainingseffekte auf analoge PTBS-Symptome gefunden: Teilnehmende, die die Anwendung funktionaler Bewertungen trainierten, zeigten beispielsweise ein geringeres Maß an negativer Reaktivität auf (z.B. bezüglich des Entsetzens über die Trauma-Filme) und weniger Intrusionen, verglichen mit Teilnehmenden aus den Kontrollbedingungen. Auch die Studie von Cheung \& Bryant (2017) nutzte eine Manipulation, bei der Teilnehmende instruiert wurden, bestimmte Bewertungen anzuwenden. Diese Bewertungen sollten jedoch angewendet werden, nachdem sich die Teilnehmenden die Trauma-Filme angesehen hatten, und sie bezogen sich spezifisch auf das Erleben von Intrusionen. Den Teilnehmenden wurde gesagt, dass Intrusionen entweder ein Zeichen für ein schlechtes psychologisches Funktionieren oder kein Zeichen für ein schlechtes psychologisches Funktionieren seien. Die Kontrollbedingung erhielt keine Anweisungen, eine bestimmte Bewertung anzuwenden. Auch wenn es nach dem Training keine Gruppenunterschiede bezüglich des Bewertungsstils gab, berichteten Teilnehmende, die die 
Kurztitel: CBM BEI PTBS - Running title: CBM FOR PTSD

Information erhalten hatten, dass Intrusionen kein Zeichen für ein schlechtes psychologisches Funktionieren seien, von weniger Intrusionen als die anderen beiden Gruppen.

In den experimentellen Studien von Woud und Kollegen (2012, 2013, 2018) wurden auch traumarelevante Bewertungsprozesse trainiert, dies erfolgte jedoch über computergesteuerte Trainings, sogenannte Cognitive Bias Modification-Appraisal (CBM-App) Trainings. Während der CBM-App Trainings wurden den Teilnehmenden eine Reihe von traumarelevanten Szenarien vorgelegt, welche von Wortfragmenten gefolgt wurden. Letztere mussten die Teilnehmenden durch das Eintippen des ersten fehlenden Buchstabens vervollständigen, und dadurch bekam das Szenario je nach Trainingsbedingung eine funktionale oder dysfunktionale Bewertung (z.B. „Meine Reaktionen seit dem Trauma zeigen, dass ich es .... ver_rbeite / verarbeite“, positives CBM-App; ,ve_dränge / verdränge“, negatives CBM-App). In den ersten beiden Studien wurde das Trauma-Film-Paradigma als analoges Trauma verwendet, einmal nach (Woud et al., 2012) und einmal vor dem CBM-App Training (Woud et al., 2013), und Bewertungen rund um das „Selbst“ trainiert. Beide Studien verglichen die Effekte eines positiven versus negativen CBMApp Trainings. Die Ergebnisse zeigten, dass das CMB-App Training zu einem trainingskongruenten Bewertungsstil führte - in beiden Studien zeigten positiv Trainierte nach dem Training einen funktionaleren Bewertungsstil als negativ trainierte Teilnehmende. Zudem berichten positiv Trainierte weniger Intrusionen (2012) beziehungsweise weniger Belastung durch ihre Intrusionen (2013) als negativ Trainierte. Das beschriebene CBM-App Training wurde durch Woud und Kollegen (Woud, Zlomuzica, et al., 2018) auch nach Re-Aktivierung eines belastenden, autobiografischen Ereignisses eingesetzt. Diese Befunde ähnelten denen der Trauma-Film-Studien: Auch hier konnte ein trainings-kongruenter Bewertungsstil induziert werden, und positiv Trainierte waren weniger durch ihre Intrusionen belastet als negativ Trainierte. Es gibt jedoch auch analoge Studien, die nicht die erwarteten Effekte finden konnten. Die Studie von Würtz et al. (2021) beispielsweise trainierte neben den Bewertungen bezüglich des Selbst auch Bewertungen bezüglich der Welt. Obwohl das positive Training einen positiveren Bewertungsstil induzierte als das negative Training, wurden keine Gruppenunterschiede z.B. bei den Intrusionen gefunden (zu ähnlichen Befunden siehe Vermeulen et al., 2019).

Gerne möchten wir nun drei klinische Studien im Rahmen dysfunktionaler Bewertungen vorstellen. Nickerson et al. (2017) untersuchte eine Stichprobe von Geflüchteten mit variierenden PTBS-Symptomen. Allen Teilnehmenden wurden traumarelevante Fotos als Analoga für traumarelevante Erinnerungen gezeigt. Bevor jedoch die Fotos gezeigt wurden, wurden die 
Kurztitel: CBM BEI PTBS - Running title: CBM FOR PTSD

Teilnehmenden in zwei Gruppen aufgeteilt. Die eine Gruppe wurde instruiert, die Fotos neu bzw. funktional zu bewerten, also als Fotos, die ihnen keinen Schaden zufügen können. Die andere Gruppe wurde instruiert, alle Gefühle, die sie beim Betrachten der Fotos haben, zu unterdrücken. Die beiden Trainings hatten keinen direkten Effekt auf PTBS-relevante Symptome, jedoch konnte gezeigt werden, dass die Gruppe, die instruiert wurde, die Fotos funktional zu bewerten und die über eine schwere PTBS-relevante Symptomatik berichteten, weniger Intrusionen erlebte. Die Studien von De Kleine et al. (2019) und Woud et al. (2021) untersuchten diagnostizierte PTBS-Patient*innen und verglichen die Effekte des schon beschriebenen positiven CBM-App Trainings mit verschiedenen Kontrollbedingungen. In De Kleine et al. (2019) beinhaltete die Kontrollbedingung, dass die Patient*innen traumarelevante Szenarien angeboten bekamen und auch ein Wortfragment vervollständigen mussten, jedoch führte letzteres nicht dazu, dass die Szenarios eine funktionale Bewertung bekamen (z.B. „Die Zukunft bringt viel ...“, positives CBM-App Training: G_tes / Gutes vs. Kontrollbedingung: verschi_denes / verschiedenes). In Woud et al. wurde eine Konzentrationsaufgabe als Kontrollbedingung eingesetzt, bei der Probanden farbig markierte Kreise abzählen und auf diese reagieren sollten. Ein weiterer Unterschied betraf die Anwendung des Trainings: In De Kleine et al. (2019) wurde das Training online durchgeführt, für die meisten Patient*innen während ihrer Wartezeit auf ihren Therapieplatz, wohingegen bei Woud et al. (2021) das Training als Add-on zur stationären Traumatherapie eingesetzt wurde. Die Ergebnisse von De Kleine et al. zeigten, dass beide Bedingungen zu einem funktionaleren Bewertungsstil und zu weniger Intrusionen führten Woud et al. hingegen fanden, dass nur Patient*innen aus der aktiven Trainingsbedingung, nicht aber aus der Kontrollbedingung weniger dysfunktionale Bewertung und PTBS-Symptome aufzeigten. In einer präventiv-orientierten Studie verglichen Pyne et al. (2019) die Effekte eines Interpretationstrainings mit einer Herzraten-Biofeedback-Intervention und einer Kontrollbedingung. Hierbei wurden US-Soldaten zu einer von drei Bedingungen eingeteilt, und sie absolvierten das jeweilige Training vor ihrem Kriegseinsatz. Im Interpretationstraining wurden ambige Szenarien zum Thema Krieg verwendet, und die Soldaten wurden instruiert, die Wortfragmente am Ende des Szenarios zu vervollständigen, wodurch die Szenarien eine neutrale bis funktionale Interpretation bekamen. Die Ergebnisse zeigten, dass das Interpretationstraining und die Biofeedback-Intervention verglichen mit der Kontrollbedingung drei Monate nach Kriegseinsatz zu weniger PTBS-Symptomen führten. Ein Jahr nach dem Kriegseinsatz war jedoch nur noch die Biofeedback-Intervention der Kontrollbedingung überlegen, während sich 
Kurztitel: CBM BEI PTBS - Running title: CBM FOR PTSD

die PTBS-relevante Symptomatik bei dem Interpretationstraining und der Kontrollbedingung nicht mehr unterschied.

Zwischenfazit: Im Bereich der experimentellen analog-Studien konnte überwiegend gezeigt werden, dass traumarelevante Bewertungen modifiziert werden können, und dass dies trainingskongruente Effekte auf (analoge) PTBS-Symptome hat. Dies scheint unabhängig von der Anwendung des Trainings zu sein, das heißt, das Training übt sowohl positive Effekte aus, wenn es computergesteuert angeboten wird (z.B. Woud et al., 2018, 2021), oder wenn funktionale Bewertungsregeln angewendet werden (Cheung \& Bryant, 2017; Schartau et al., 2009). Im Bereich der klinischen Anwendung gibt es erste vielversprechenden Befunde (z.B. De Kleine et al., 2019; Nickerson et al., 2017; Woud et al., 2021) im Bereich von Bewertungs- sowie Interpretationsprozessen, diese Ergebnisse müssen jedoch durch zukünftige Forschung repliziert und spezifiziert werden.

\section{Modifikation von Gedächtnisprozessen}

Nach unserem aktuellen Wissen gibt es zurzeit drei publizierte Trainings, die darauf abzielen, Gedächtnisprozesse zu verändern. Zwei davon verwendeten ein sogenanntes „Gedächtnisspezifitätstraining“ (Memory Specificity Training, MeST; Raes et al., 2009). In diesem Training werden Teilnehmende trainiert, spezifische Erinnerungen besser und detailreicher abzurufen. Motiviert wurde dies durch Studien, die zeigen konnten, dass die PTBS von einem übergeneralisierten, autobiografischen Gedächtnis gekennzeichnet ist (z.B. Kleim \& Ehlers, 2008; McNally et al., 1994). Das MeST beinhaltet vier einstündige, wöchentliche Gruppensitzungen, in denen Teilnehmende trainiert werden, spezifische Erinnerungen nach der Präsentation eines Hinweisworts abzurufen und möglichst viele Details dieser Erinnerung zu beschreiben. Zwischen den Sitzungen erhalten die Teilnehmenden Hausaufgaben, um dies weiter zu trainieren. $\mathrm{Zu}$ Beginn des Trainings wird der Fokus auf positive und neutrale Erinnerungen gelegt, im weiteren Verlauf werden auch negative Erinnerungen mit aufgenommen. In einer ersten Pilotstudie wurden die Effekte von MeST in einer Stichprobe iranischer Veteranen getestet (Moradi et al., 2014). Der Autobiographical Memory Test (AMT; Williams \& Broadbent, 1986) wurde eingesetzt, um zu überprüfen, ob das Training zu einer Zunahme der Gedächtnisspezifität führte. Die Ergebnisse im AMT zeigten, dass Teilnehmende in der MeST-Bedingung, im Vergleich zur Kontrollbedingung, sowohl direkt nach dem Training als auch im 3-MonatsFollow-Up einen besseren Abruf spezifischer autobiografischer Erinnerungen aufwiesen. 
Kurztitel: CBM BEI PTBS - Running title: CBM FOR PTSD

Außerdem zeigten die Teilnehmenden in der MeST-Bedingung sowohl direkt danach als auch im 3-Monats-Follow-Up eine Verbesserung ihrer Gedächtnisspezifität im Vergleich zum Beginn des Trainings, was jedoch nicht bei der Kontrollbedingung gefunden wurde. Bezüglich der PTBSrelevanten Symptomatik zeigten beide Gruppen im Laufe der Studie eine Reduktion bei den Symptomgruppen Vermeidung und Intrusionen. In der Studie von Maxwell et al. (2016) wurde MeST bei diagnostizierten PTBS-Patient*innen mit Cognitive Processing Therapy (CPT; Resick et al., 2010) verglichen. Das MeST beinhaltete sechs Sitzungen, die CPT erstreckte sich über 12 Wochen. Nach den jeweiligen Interventionen zeigten beide Gruppen eine Verbesserung im Abruf spezifischer autobiografischer Erinnerungen (gemessen mit der AMT). Außerdem konnte bei beiden Gruppen eine Verbesserung der PTBS- und der depressiven Symptome sowie in der allgemeinen Funktionsfähigkeit verzeichnet werden. In dem aktuellen RCT von Moradi et al. (2021) wurde das MeST weiter spezifiziert und beinhaltete ein Training, das die Flexibilität des autobiografischen Gedächtnisses verbessern sollte (Memory Flexibility Training, MemFlex). Die zugrundeliegende Idee dieser Spezifizierung war, dass es auch hilfreich sein könnte, wenn Patienten trainiert werden, flexibler von einem zum anderen Ereignis wechseln zu können. Die Stichprobe bestand aus diagnostizierten PTBS-Patient*innen und das MemFlex wurde mit einer Wartekontrollgruppe verglichen. Die Ergebnisse zeigten, dass das MemFlex-Training, verglichen mit der Wartekontrollgruppe, zu einem verbesserten Abruf spezifischer autobiografischer Erinnerungen führte. Zudem berichteten Patient*innen des MemFlex-Trainings im Anschluss von weniger PTBS-Symptomen.

Zwischenfazit: Trotz der ersten vielversprechenden Befunde im Bereich der Gedächtnistrainings sollten diese Befunde vorsichtig interpretiert werden, da es sich unter anderem um Pilotstudien mit kleinen Stichproben handelt (Moradi et al., 2014). Wichtige Ansatzpunkte für die zukünftige Forschung wären somit klinische Studien mit größeren Stichproben und adäquaten Kontrollbedingungen. Ein anderer wichtiger Punkt bezieht sich auf die zugrundeliegenden Mechanismen von MeST, hier zeigten Ergebnisse von Moradi et al. (2014), dass MeST nicht zu einer Reduzierung der kriegsbezogenen Erinnerungen geführt hat, und dieses Ergebnis sollte durch Nachfolgeuntersuchungen besser verstanden werden.

\section{Zusammenfassung und Ausblick für die weitere Forschung}

Cognitive Bias Modification (CBM) ist ein junges Forschungsfeld, jedoch gibt es auf dem Gebiet der PTBS eine Reihe von vielversprechenden Ergebnissen. Im Bereich der Aufmerksamkeit 
wurden vor allem klinische Studien durchgeführt, welche zum größten Teil auch Trainingseffekte auf (analoge) PTBS-Symptome gefunden haben. Diese Studien zeigten jedoch auch, dass die Symptomreduktion nicht immer mit einer Reduktion der Aufmerksamkeitsverzerrungen einherging. Einerseits könnte man nun argumentieren, dass dies nicht der zentrale Aspekt ist, da aus klinischer Perspektive die Symptomveränderung im Mittelpunkt stehen sollte. Andererseits kann man CBM-Trainings nur systematisch verbessern, wenn man die zugrundliegenden Mechanismen versteht. Dies wird vor allem deutlich, wenn man sich die Entwicklungen bezüglich der Kontrollbedingungen in diesem Bereich vor Augen führt - was ursprünglich als nicht-aktive Kontrollbedingung begann, reduzierte in den untersuchten klinischen Stichproben dennoch PTBS-Symptome, und wurde somit im Laufe der Zeit zur aktiven Kontrollbedingung, z.B. im Bereich der Aufmerksamkeitskontrolltrainings, mit dementsprechend neuen Hypothesen bezüglich der Trainingsmechanismen (siehe Badura-Brack et al., 2015; Lazarov et al., 2019) (zu einer Diskussion zu Kontrollbedingungen bei CBM siehe Blackwell et al., 2017). Im Rahmen von Bewertungs- und Interpretationstrainings haben experimentelle sowie klinische Studien einige vielversprechende Befunde hervorgebracht (De Kleine et al., 2019; Nickerson et al., 2017; Schartau et al., 2009; Woud et al., 2021), und diese ersten Ergebnisse stimmen hoffnungsvoll bezüglich des therapeutischen Potenzials solcher Trainings. Im Bereich des Gedächtnisses gibt es bis jetzt nur wenige Trainingsstudien, die auch mit verschiedenen Einschränkungen einhergehen, dennoch sind wir vorsichtig optimistísch, was diese Trainings angeht.

Aus der Forschungsperspektive sind nun verschiedenste Aspekte relevant, einer betrifft die Rolle der Konfrontation als möglicher Wirkfaktor. Alle CBM-Trainings enthalten störungsrelevante Reize, z.B. Fotos von bedrohlichen Situationen oder dysfunktionale Gedanken. Obwohl diese Reize während des Trainings systematisch mit einer funktionalen Reaktion kombiniert werden, werden die Teilnehmenden in erster Instanz auch mit diesen Reizen und ihren Reaktionen darauf konfrontiert. Dies allein könnte schon dazu führen, dass bestimmte Symptome abnehmen und dass Patient*innen lernen, besser mit ihren Reaktionen auf diese Reize umzugehen, und zukünftige Studien sollten dies genauer abklären. Zudem wäre es ratsam, holistischere Ansätze zu verfolgen, indem die Effekte des Trainings beispielsweise auch auf psychophysiologische Korrelate untersucht werden. Dies erscheint vor allem sinnvoll, wenn man an das E-Kriterium der PTBS, Veränderungen des Erregungsniveaus und der Reaktivität, denkt (DSM-5; American Psychiatric Association, 2013). 
Kurztitel: CBM BEI PTBS - Running title: CBM FOR PTSD

\section{Klinisch relevante Aspekte}

Im Folgenden möchten wir Aspekte diskutieren, die vor allem aus klinischer Sicht relevant sind. Ein erster wichtiger Aspekt ist der Transfer - hier gibt es noch zu wenig Befunde, die robust und systematisch zeigen können, dass CBM-Trainings (analoge) PTBS-Symptome reduzieren. Die Frage, inwieweit CBM-Trainings auch positive Effekte auf die Symptome einer komplexen PTBS (ICD-11; World Health Organization, 2019), beispielsweise auf das negative Selbstbild, ausüben, ist bislang ungeklärt und wäre ein weiterer wichtiger Aspekt für die zukünftige Forschung. Basierend auf den ersten vielversprechenden Befunden zu Bewertungstrainings (De Kleine et al., 2019; Woud et al., 2021) könnten hierzu spezifische Nachfolgestudien eingesetzt und untersucht werden, inwieweit ein Bewertungstraining zur Modifikation des negativen Selbstbildes als Add-On positive Effekte aufzeigt. Ein weiterer Aspekt betrifft die Langzeiteffekte von CBM auf kognitiver sowie Symptomebene. Die Studie von Pyne et al. (2019), welche ein Interpretationstraining mit darauffolgender Ein-JahresKatamnese beinhaltete, ist hier leider die Ausnahme, und somit wäre es wünschenswert, dass zukünftige und adäquat-gepowerte Studien sich diesen Effekten widmen. Die Frage, wann CBM am besten als Intervention eingesetzt werden kann, ist ein weiterer wichtiger Aspekt. Die präsentierten Studien haben CBM unter anderem als Prävention (z.B. Wald et al., 2016) und als zusätzliche (z.B. Woud et al., 2021) sowie eigenständige Intervention (z.B. Moradi et al., 2014) angewendet. Die meisten Studien verbuchten positive Effekte, so zeigten aber beispielsweise die Patient*innen nach dem MEST weiterhin ein Symptomlevel über den klinischen Schwellenwerten einer PTBS auf. Dies legt nahe, dass MEST als alleinige Behandlungsmethode für PTBS unzureichend ist, und es ist anzunehmen, dass dies auch für andere CBMTrainingsbereiche gilt. Zukünftige Forschung sollte somit klären, unter welchen Bedingungen CBM am besten implementiert werden kann. Es ist nicht zu erwarten bzw. wünschenswert, dass CBM jemals eine Psychotherapie ersetzen kann, es sollte eher als Mechanismus-spezifisches Add-on betrachtet werden. Die derzeitige Empfehlung wäre somit, CBM unterstützend im ambulanten oder stationären Setting einzusetzen, oder als Hausaufgabe zwischen den Sitzungen, beispielsweise im Rahmen der kognitiven Umstrukturierung, um dadurch die Therapieeffekte und Transfer dieser Effekte in den Alltag der Patient*innen weiter zu verstärken (für eine aktuelle Übersichtsarbeit s. Michael et al., 2019). Denkbar wäre auch, dass CBM als niedrigschwellige, erste Intervention in der Wartezeit auf einen Therapieplatz eingesetzt werden kann, oder als Rezidivprophylaxe. Zudem ist es relevant, besser zu verstehen, welche Art von Training mit 
Kurztitel: CBM BEI PTBS - Running title: CBM FOR PTSD

welchen Reizen für wen hilfreich ist. Individualisierte Angebote wären in Zukunft denkbar - so könnten Trainings zur Modifikation von dysfunktionalen Bewertungen vor allem hilfreich im Bereich der multiplen, interpersonellen Traumatisierung sein, bei der (chronische) dysfunktionale Bewertungen stärker manifestiert sind als beispielweise nach einer PTBS durch einen Überfall. Bei einem Überfall wiederrum wäre möglicherweise ein Aufmerksamkeitstraining, bei dem man die Aufmerksamkeit hin zu bedrohlichen Reizen trainiert, sinnvoll, um dadurch die erhöhte Wachsamkeit zu reduzieren.

Neben der klassischen CBM-Forschung gibt es weitere kognitive Trainings, die im Kontext von (analogen) Traumata und (analogen) PTBS-Symptomen eingesetzt werden. Diese Trainings sind jedoch näher an kognitiv-neurowissenschaftliche Modelle der PTBS angelehnt. Ein Beispiel hierfür ist der Einsatz räumlich-visueller Aufgaben (das Computerspiel „Tetris“) direkt nach einem Trauma (z.B. Iyadurai et al., 2018) oder nach der Reaktivierung des Traumas im Rahmen der Traumatherapie (z.B. Kessler et al., 2018). Die Trainings haben zum Ziel, mit der Konsolidierung sensorischer Aspekte des Traumas zu interferieren, um damit vor allem das Aufkommen von Intrusionen zu verhindern bzw. zu verringern. Bis jetzt gibt es erste vielversprechende Befunde, es bedarf jedoch, ähnlich wie im Forschungsbereich der CBM, weiterer Nachfolge- und Längsschnittstudien, um die Effekte dieser Trainings besser zu verstehen.

\section{Konklusion}

In unserer Übersicht konnten wir zeigen, dass es im Rahmen der CBM-Forschung im Bereich von (analogen) Traumata und der PTBS im experimentellen sowie klinischen Bereich erste vielversprechende Befunde gibt. Generell bedarf es jedoch systematischer Nachfolgeuntersuchungen, um sowohl die zugrundeliegenden Mechanismen als auch die klinische Effektivität von CBM besser zu verstehen. Wir hoffen, dass dieser Artikel viele Kolleg*innen motiviert, sich diesen spannenden Forschungsfragen zu widmen. 
Kurztitel: CBM BEI PTBS - Running title: CBM FOR PTSD

\section{Conflict of Interest Statement}

Die Autorinnen und Autoren haben keine Interessenskonflikte zu berichten.

\section{Funding Sources}

Marcella Woud wird durch eine Sachbeihilfe der DFG (WO 2018/2-1), dem Emmy Noether Programm der DFG (WO 2018/3-1) und dem SFB 1280 „Extinktionslernen“ der DFG unterstützt. Charlotte Wittekind wird von einer Sachbeihilfe der DFG (WI 4856/3-1) und einem Stipendium der Daimler und Benz Stiftung (32-12/18) unterstützt. Felix Würtz wird durch ein Promotionsstipendium der Studienstiftung des deutschen Volkes unterstützt. Die finanzierenden Trägerschaften waren nicht an der Erstellung des Manuskripts beteiligt.

\section{Author Contributions}

Marcella L. Woud: Konzeptualisierung, Verfassen des ersten Entwurfs, Bearbeitung und Editierung, Literaturzusammenstellung

Charlotte E. Wittekind: Verfassen des ersten Entwurfs, Bearbeitung und Editierung, Literaturzusammenstellung

Felix Würtz: Konzeptualisierung, Verfassen des ersten Entwurfs, Bearbeitung und Editierung, Literaturzusammenstellung 
Kurztitel: CBM BEI PTBS - Running title: CBM FOR PTSD

\section{Literaturverzeichnis}

American Psychiatric Association (Ed.). (2013). Diagnostic and Statistical Manual of Mental Disorders: DSM-5 (5th ed). American Psychiatric Association.

Badura-Brack, A. S., Naim, R., Ryan, T. J., Levy, O., Abend, R., Khanna, M. M., McDermott, T. J., Pine, D. S., \& Bar-Haim, Y. (2015). Effect of Attention Training on Attention Bias Variability and PTSD Symptoms: Randomized Controlled Trials in Israeli and U.S. Combat Veterans. American Journal of Psychiatry, 172(12), 1233-1241. https://doi.org/10.1176/appi.ajp.2015.14121578

Blackwell, S. E., \& Woud, M. L. (2022). Making the leap: From experimental psychopathology to clinical trials. Journal of Experimental Psychopathology, 13(1), 204380872210800. https://doi.org/10.1177/20438087221080075

Blackwell, S. E., Woud, M. L., \& MacLeod, C. (2017). A Question of Control? Examining the Role of Control Conditions in Experimental Psychopathology using the Example of Cognitive Bias Modification Research. The Spanish Journal of Psychology, 20, E54. https://doi.org/10.1017/sjp.2017.41

Bomyea, J., Johnson, A., \& Lang, A. J. (2017). Information Processing in PTSD: Evidence for Biased Attentional, Interpretation, and Memory Processes. Psychopathology Review, a4(3), 218-243. https://doi.org/10.5127/pr.037214

Brewin, C. R. (2014). Episodic memory, perceptual memory, and their interaction: Foundations for a theory of posttraumatic stress disorder. Psychological Bulletin, 140(1), 69-97. https://doi.org/10.1037/a0033722

Bryant, R. A., \& Guthrie, R. M. (2007). Maladaptive self-appraisals before trauma exposure predict posttraumatic stress disorder. Journal of Consulting and Clinical Psychology, 75(5), 812-815. https://doi.org/10.1037/0022-006X.75.5.812 
Kurztitel: CBM BEI PTBS - Running title: CBM FOR PTSD

Cheung, J., \& Bryant, R. A. (2017). The impact of appraisals on intrusive memories. Journal of Behavior Therapy and Experimental Psychiatry, 54, 108-111. https://doi.org/10.1016/j.jbtep.2016.07.005

Clarke, P. J., Notebaert, L., \& MacLeod, C. (2014). Absence of evidence or evidence of absence: Reflecting on therapeutic implementations of attentional bias modification. $B M C$ Psychiatry, 14(1), 8. https://doi.org/10.1186/1471-244X-14-8

Dalgleish, T. (2004). Cognitive Approaches to Posttraumatic Stress Disorder: The Evolution of Multirepresentational Theorizing. Psychological Bulletin, 130(2),228-260. https://doi.org/10.1037/0033-2909.130.2.228

De Kleine, R. A., Woud, M. L., Ferentzi, H., Hendriks, G.-J., Broekman, T. G., Becker, E. S., \& Van Minnen, A. (2019). Appraisal-based cognitive bias modification in patients with posttraumatic stress disorder: A randomised clinical trial. European Journal of Psychotraumatology, 10(1), 1625690. https://doi.org/10.1080/20008198.2019.1625690

Ehlers, A., \& Clark, D. M. (2000). A cognitive model of posttraumatic stress disorder. Behaviour Research and Therapy, 38(4), 319-345. https://doi.org/10.1016/S0005-7967(99)00123-0

Foa, E. B., Steketee, G., \& Rothbaum, B. O. (1989). Behavioral/cognitive conceptualizations of post-traumatic stress disorder. Behavior Therapy, 20(2), 155-176. https://doi.org/10.1016/S0005-7894(89)80067-X

Fodor, L. A., Georgéscu, R., Cuijpers, P., Szamoskozi, Ş., David, D., Furukawa, T. A., \& Cristea, I. A. (2020). Efficacy of cognitive bias modification interventions in anxiety and depressive disorders: A systematic review and network meta-analysis. The Lancet Psychiatry, 7(6), 506-514. https://doi.org/10.1016/S2215-0366(20)30130-9 
Kurztitel: CBM BEI PTBS - Running title: CBM FOR PTSD

Gober, C. D., Lazarov, A., \& Bar-Haim, Y. (2021). From cognitive targets to symptom reduction: Overview of attention and interpretation bias modification research. Evidence Based Mental Health, 24(1), 42-46. https://doi.org/10.1136/ebmental-2020-300216

Horsch, A., Vial, Y., Favrod, C., Harari, M. M., Blackwell, S. E., Watson, P., Iyadurai, L., Bonsall, M. B., \& Holmes, E. A. (2017). Reducing intrusive traumatic memories after emergency caesarean section: A proof-of-principle randomized controlled study.

Behaviour Research and Therapy, 94, 36-47. https://doi.org/10.1016/j.brat.2017.03.018

Imbriano, G., Waszczuk, M., Rajaram, S., Ruggero, C., Miao, J., Clouston, S., Luft, B., Kotov, R., \& Mohanty, A. (2022). Association of attention and memory biases for negative stimuli with post-traumatic stress disorder symptoms. Journal of Anxiety Disorders, 85, 102509. https://doi.org/10.1016/j.janxdis.2021.102509

Iyadurai, L., Blackwell, S. E., Meiser-Stedman, R., Watson, P. C., Bonsall, M. B., Geddes, J. R., Nobre, A. C., \& Holmes, E. A. (2018). Preventing intrusive memories after trauma via a brief intervention involving Tetris computer game play in the emergency department: A proof-of-concept randomized controlled trial. Molecular Psychiatry, 23(3), 674-682. https://doi.org/10.1038/mp.2017.23

Jones, E. B., \& Sharpe, L. (2017). Cognitive bias modification: A review of meta-analyses. Journal of Affective Disorders, 223, 175-183. https://doi.org/10.1016/j.jad.2017.07.034 Kessler, H., Holmes, E. A., Blackwell, S. E., Schmidt, A.-C., Schweer, J. M., Bücker, A., Herpertz, S., Axmacher, N., \& Kehyayan, A. (2018). Reducing intrusive memories of trauma using a visuospatial interference intervention with inpatients with posttraumatic stress disorder (PTSD). Journal of Consulting and Clinical Psychology, 86(12), 10761090. https://doi.org/10.1037/ccp0000340 
Kurztitel: CBM BEI PTBS - Running title: CBM FOR PTSD

Khanna, M. M., Badura-Brack, A. S., McDermott, T. J., Shepherd, A., Heinrichs-Graham, E., Pine, D. S., Bar-Haim, Y., \& Wilson, T. W. (2016). Attention training normalises combatrelated post-traumatic stress disorder effects on emotional Stroop performance using lexically matched word lists. Cognition and Emotion, 30(8), 1521-1528. https://doi.org/10.1080/02699931.2015.1076769

Kleim, B., \& Ehlers, A. (2008). Reduced autobiographical memory specificity predicts depression and posttraumatic stress disorder after recent trauma. Journal of Consulting and Clinical Psychology, 76(2), 231-242. https://doi.org/10.1037/0022-006X.76.2.231

Koster, E. H. W., Fox, E., \& MacLeod, C. (2009). Introduction to the special section on cognitive bias modification in emotional disorders. Journal of Abnormal Psychology, 118(1), 1-4. https://doi.org/10.1037/a0014379

Kuckertz, J. M., Amir, N., Boffa, J. W., Warren, C. K., Rindt, S. E. M., Norman, S., Ram, V., Ziajko, L., Webb-Murphy, J., \& McLay, R. (2014). The effectiveness of an attention bias modification program as an adjunctive treatment for Post-Traumatic Stress Disorder. Behaviour Research and Therapy, 63, 25-35. https://doi.org/10.1016/j.brat.2014.09.002 Lazarov, A., Suarez-Jimenez, B., Abend, R., Naim, R., Shvil, E., Helpman, L., Zhu, X., Papini, S., Duroski, A., Rom, R., Schneier, F. R., Pine, D. S., Bar-Haim, Y., \& Neria, Y. (2019). Bias-contingent attention bias modification and attention control training in treatment of PTSD: A randomized control trial. Psychological Medicine, 49(14), 2432-2440. https://doi.org/10.1017/S0033291718003367

MacLeod, C., \& Mathews, A. (2012). Cognitive Bias Modification Approaches to Anxiety. Annual Review of Clinical Psychology, 8(1), 189-217. https://doi.org/10.1146/annurevclinpsy-032511-143052 
Kurztitel: CBM BEI PTBS - Running title: CBM FOR PTSD

MacLeod, C., Mathews, A., \& Tata, P. (1986). Attentional bias in emotional disorders. Journal of Abnormal Psychology, 95(1), 15-20. https://doi.org/10.1037/0021-843X.95.1.15

Mathews, A., \& MacLeod, C. (2005). Cognitive Vulnerability to Emotional Disorders. Annual Review of Clinical Psychology, 1(1), 167-195. https://doi.org/10.1146/annurev.clinpsy.1.102803.143916

Maxwell, K., Callahan, J. L., Holtz, P., Janis, B. M., Gerber, M. M., \& Connor, D. R. (2016). Comparative study of group treatments for posttraumatic stress disorder. Psychotherapy, 53(4), 433-445. https://doi.org/10.1037/pst0000032

McNally, R. J., Litz, B. T., Prassas, A., Shin, L. M., \& Weathers, F. W. (1994). Emotional priming of autobiographical memory in post-traumatic stress disorder. Cognition \& Emotion, 8(4), 351-367. https://doi.org/10.1080/02699939408408946

Michael, T., Schanz, C. G., Mattheus, H. K., Issler, T., Frommberger, U., Köllner, V., \& Equit, M. (2019). Do adjuvant interventions improve treatment outcome in adult patients with posttraumatic stress disorder receiving trauma-focused psychotherapy? A systematic review. European Journal of Psychotraumatology, 10(1), 1634938. https://doi.org/10.1080/20008198.2019.1634938

Moradi, A. R., Moshirpanahi, S., Parhon, H., Mirzaei, J., Dalgleish, T., \& Jobson, L. (2014). A pilot randomized controlled trial investigating the efficacy of MEmory Specificity Training in improving symptoms of posttraumatic stress disorder. Behaviour Research and Therapy, 56, 68-74. https://doi.org/10.1016/j.brat.2014.03.002

Moradi, A. R., Piltan, M., Choobin, M. H., Azadfallah, P., Watson, P., Dalgleish, T., \& Hitchcock, C. (2021). Proof of Concept for the Autobiographical Memory Flexibility (MemFlex) Intervention for Posttraumatic Stress Disorder. Clinical Psychological Science, 9(4), 686-698. https://doi.org/10.1177/2167702620982576 
Kurztitel: CBM BEI PTBS - Running title: CBM FOR PTSD

Nickerson, A., Garber, B., Liddell, B. J., Litz, B. T., Hofmann, S. G., Asnaani, A., Ahmed, O., Cheung, J., Huynh, L., Pajak, R., \& Bryant, R. A. (2017). Impact of Cognitive Reappraisal on Negative Affect, Heart Rate, and Intrusive Memories in Traumatized Refugees. Clinical Psychological Science, 5(3), 497-512. https://doi.org/10.1177/2167702617690857

Posner, M. I. (1980). Orienting of Attention. Quarterly Journal of Experimental Psychology, 32(1), 3-25. https://doi.org/10.1080/00335558008248231

Pyne, J. M., Constans, J. I., Nanney, J. T., Wiederhold, M. D., Gibson, D.P., Kimbrell, T., Kramer, T. L., Pitcock, J. A., Han, X., Williams, D. K., Chartrand, D., Gevirtz, R. N., Spira, J., Wiederhold, B. K., McCraty, R., \& McCune, T. R. (2019). Heart Rate Variability and Cognitive Bias Feedback Interventions to Prevent Post-deployment PTSD: Results from a Randomized Controlled Trial. Military Medicine, 184(1-2), e124-e132. https://doi.org/10.1093/milmed/usy171

Raes, F., Williams, J. M. G., \& Hermans, D. (2009). Reducing cognitive vulnerability to depression: A preliminary investigation of MEmory Specificity Training (MEST) in inpatients with depressive'symptomatology. Journal of Behavior Therapy and Experimental Psychiatry, 40(1), 24-38. https://doi.org/10.1016/j.jbtep.2008.03.001

Resick, P. A., Monson, C. M., \& Chard, K. M. (2010). Cognitive processing therapy: Veteran/military version. Washington, DC: Department of Veterans’ Affairs.

Schartau, P. E. S., Dalgleish, T., \& Dunn, B. D. (2009). Seeing the bigger picture: Training in perspective broadening reduces self-reported affect and psychophysiological response to distressing films and autobiographical memories. Journal of Abnormal Psychology, 118(1), 15-27. https://doi.org/10.1037/a0012906 
Kurztitel: CBM BEI PTBS - Running title: CBM FOR PTSD

Schoorl, M., Putman, P., \& Van Der Does, W. (2013). Attentional Bias Modification in Posttraumatic Stress Disorder: A Randomized Controlled Trial. Psychotherapy and Psychosomatics, 82(2), 99-105. https://doi.org/10.1159/000341920

Segal, A., Wald, I., Pine, D. S., Halpern, P., \& Bar-Haim, Y. (2020). Attention control therapy for acute stress disorder: A randomized controlled trial. Depression and Anxiety, 37(10), 1017-1025. https://doi.org/10.1002/da.23040

Vermeulen, M., Brown, A. D., Raes, F., \& Krans, J. (2019). Decreasing Event Centrality in Undergraduates Using Cognitive Bias Modification of Appraisals. Cognitive Therapy and Research, 43(1), 214-225. https://doi.org/10.1007/s10608-018-9936-3

Verwoerd, J., Wessel, I., \& de Jong, P. J. (2012). Fewer intrusions after an attentional bias modification training for perceptual reminders of analogue trauma. Cognition \& Emotion, 26(1), 153-165. https://doi.org/10.1080/02699931.2011.563521

Wald, I., Bitton, S., Levi, O., Zusmanovich, S., Fruchter, E., Ginat, K., Charney, D. S., Pine, D. S., \& Bar-Haim, Y. (2017). Acute delivery of attention bias modification training $(\mathrm{ABMT})$ moderates the association between combat exposure and posttraumatic symptoms: A feasibility study. Biological Psychology, 122, 93-97. https://doi.org/10.1016/j.biopsycho.2016.01.005

Wald, I., Fruchter, E., Ginat, K., Stolin, E., Dagan, D., Bliese, P. D., Quartana, P. J., Sipos, M. L., Pine, D. S., \& Bar-Haim, Y. (2016). Selective prevention of combat-related posttraumatic stress disorder using attention bias modification training: A randomized controlled trial. Psychological Medicine, 46(12), 2627-2636. https://doi.org/10.1017/S0033291716000945

Wald, I., Shechner, T., Bitton, S., Holoshitz, Y., Charney, D. S., Muller, D., Fox, N. A., Pine, D. S., \& Bar-Haim, Y. (2011). Attention bias away from threat during life threatening danger 
Kurztitel: CBM BEI PTBS - Running title: CBM FOR PTSD

predicts PTSD symptoms at one-year follow-up. Depression and Anxiety, 28(5), 406-411. https://doi.org/10.1002/da.20808

Williams, J. M., \& Broadbent, K. (1986). Autobiographical memory in suicide attempters. Journal of Abnormal Psychology, 95(2), 144-149. https://doi.org/10.1037/0021843X.95.2.144

World Health Organization. (2019). International statistical classification of diseases and related health problems (11th ed.). https://icd.who.int/

Woud, M. L., \& Becker, E. S. (2014). Editorial for the Special Issue on Cognitive Bias Modification Techniques: An Introduction to a Time Traveller's Tale. Cognitive Therapy and Research, 38(2), 83-88. https://doi.org/10.1007/s10608-014-9605-0

Woud, M. L., Blackwell, S. E., Shkreli, L., Würtz, F., Cwik, J. C., Margraf, J., Holmes, E. A., Steudte-Schmiedgen, S., Herpertz, S., \& Kessler, H. (2021). The Effects of Modifying Dysfunctional Appraisals in Posttraumatic Stress Disorder Using a Form of Cognitive Bias Modification: Results of a Randomized Controlled Trial in an Inpatient Setting. Psychotherapy and Psychosomatics, 1-17. https://doi.org/10.1159/000514166

Woud, M. L., Holmes, E. A., Postma, P., Dalgleish, T., \& Mackintosh, B. (2012). Ameliorating intrusive memories of distressing experiences using computerized reappraisal training. Emotion, 12(4), 778-784. https://doi.org/10.1037/a0024992

Woud, M. L., Postma, P., Holmes, E. A., \& Mackintosh, B. (2013). Reducing analogue trauma symptoms by computerized reappraisal training - Considering a cognitive prophylaxis? Journal of Behavior Therapy and Experimental Psychiatry, 44(3), 312-315. https://doi.org/10.1016/j.jbtep.2013.01.003 
Kurztitel: CBM BEI PTBS - Running title: CBM FOR PTSD

Woud, M. L., Verwoerd, J., \& Krans, J. (2017). Modification of cognitive biases related to posttraumatic stress: A systematic review and research agenda. Clinical Psychology Review, 54, 81-95. https://doi.org/10.1016/j.cpr.2017.04.003

Woud, M. L., Zlomuzica, A., Cwik, J. C., Margraf, J., Shkreli, L., Blackwell, S. E., Gladwin, T. E., \& Ehring, T. (2018). Effects of appraisal training on responses to a distressing autobiographical event. Journal of Anxiety Disorders, 56, 26-34. https://doi.org/10.1016/j.janxdis.2018.03.010

Würtz, F., Krans, J., Blackwell, S. E., Cwik, J. C., Margraf, J., \& Woud, M. L. (2021). Using Cognitive Bias Modification-Appraisal Training to Manipulate Appraisals about the Self and the World in Analog Trauma. Cognitive Therapy and Research.

https://doi.org/10.1007/s10608-021-10257-x 